Anatomical Science (2018) Volume 1

doi:10.24294/as.v1i3.969

\title{
Content Based Medical Image Retrieval System Based on Gradient Orientation and Edge Information
}

\section{Pranjit Das}

Department of CSE, National Institute of Technology Nagaland, Dimapur, India

\begin{abstract}
Retrieval of biomedical pictures is a significant side of computer based diagnosis. It helps the radiologist and restorative authority to spot and analyze the particular disease. This paper proposes a Content Based Medical Image Retrieval (CBMIR) approach for retrieving similar biomedical images. The extraction of retrieving features is based on histogram of oriented gradients (HOG) and canny edge detection. To reduce the dimensionality, principal component analysis(PCA) is employed over the feature vector. The experiments are conducted on high-resolution computed tomography medical images of lungs. With the average retrieval rate (ARR) and average retrieval precision (ARP), the performance of the proposed approach is analyzed and compared with other existing methods viz. Local Binary Pattern (LBP), LBP with uniform patterns (LBPu2), Local Mesh Pattern with uniform patterns (LMePu2) and LMeP with gabor transform (GLMeP).
\end{abstract}

Keywords: Biomedical; Image Retrieval; HOG; PCA

\section{Introduction}

Nowadays the amount of visual and multimedia data is raising at a huge quantity which leads to creation of large digital databases and the simple text based queries are not so effective for such a huge database. The imaging technology does a crucial function in the field of medical science and becomes a very popular efficient technology. In 1895, Wilhelm Conrad Roentgen ${ }^{[1]}$ started the medical imaging by discovering the X-ray and then nuclear medicine was introduced in 1950 which is a combination of radionuclides with radiopharmaceuticals. After that John Wild used the ultrasound for the first time in the medical field to compute the thickness of bowel tissue $^{[2,3]}$. Computer tomography (CT) was then adopted in medical science and then, Sir Godfrey Hounsfield invented the first commercial CT scanner in 1967. Later, many well-known techniques are included in medical imaging like ultrasonography, electrography, endoscopy, $\mathrm{PACS}^{[4-6]}$, magnetic resonance imaging (MRI) and many more.

The number of medical diagnostic test in the diagnosis centers or hospitals is increasing in terribly high rate day by day. Most of the scanning based diagnostic test uses the biomedical imaging technology.
Because of the speedy use of biomedical images within the hospitals, an outsized form of medical pictures is generated every day. Medical imaging performs an important role in each medical setting and in any respect levels of healthcare. It helps the physicians to hit a lot of correct diagnoses and applicable treatment selections. Diagnosis and treatment in digital health are often terribly troublesome to attain with any level of accuracy without biomedical imaging.

From the last decades, the retrieval of biomedical images become the one of the most active research areas in the field of medical imaging and computer vision. Doctors are supported by image retrieval systems in retrieving the similar images and similar past cases to gain the detailed knowledge of the patient's injury or disease standing. The radiologist also gets assist to prepare or produce particular medical diagnosis report in less time. Apart from diagnostics, the medical image retrieval system also give assistance in research and teaching. The main aim of image retrieval is to determine the related images. Initially, text based image retrieval systems are proposed for retrieving the related images that is based on text query also known as concept based or description based image retrieval. With the textual 
query, the similar images are retrieved in the concept based image retrieval systems. But these systems are not become so effective in finding out the similar images. Later a new form of image retrieval is introduced with the visual content of images and is known as content-based image retrieval (CBIR). This is also known as content-based visual information retrieval or query by image content.

In the Nineties at first, the medical pictures retrieval is within the traditional retrieval system based on content but it is not so efficient. Afterward, CBMIR is presented and many systems are introduced as ASSERT $^{[7]}$, IRMA $^{[8]}$, BRICS ${ }^{[9]}$, FIRE $^{[10]}$ and many more. Several review articles ${ }^{[11-19]}$ explicate the existing state-of-the-art techniques or methodologies on biomedical image retrievals.

\section{Review on existing methods}

This section presents several existing state-of-the-art CBMIR systems and discuss about the different methodologies used by various CBMIR techniques.

For medical image retrieval, researchers concentrate on binary descriptors and barcode is one amongst them. The binarization of radon projection results in the formation of radon barcode (RBC) and by collecting, a vector is generated known as "barcode" which is introduced by Tizhoosh [20,21]. Tizhoosh proposed barcode annotation for CBMIR systems. Local binary pattern and local radon binary pattern are also implanted for medical image retrieval. Later the MinMax radon barcodes ${ }^{[22]}$ is introduced to overcome the disadvantages of radon barcode that is many unique contents may lose by randon barcode and the local thresholding method doesn't cover the curvature of projection. Based on the barcodes some other methodologies ${ }^{[23-25]}$ are also introduced.

Fuzzy and neural network based concepts are adopted by many CBMIR systems. Recently, pulse coupled neural network and non-subsampled contourlet transform based CBMIR method is introduced by Kundu et al. ${ }^{[26]}$. Ma et al. ${ }^{[27]}$ propose a new CBMIR method know as fused context-sensitive similarity (FCSS) based on support vector machine (SVM) and several distance measures. vector quantization with fuzzy signatures is used in ${ }^{[28]}$ to develop a new system called fuzzy medical image retrieval (FMIR). In $^{[29,30]}$, compact composite descriptor (CCD) is employed to proposed a retrieval system for radiology images which is fuzzy rule-based system. Recently deep leaning from the machine learning techniques performs a crucial role in the computer vision. Various works ${ }^{[31-38]}$ of visual pattern recognition used the concept of convolution neural network $(\mathrm{CNN})$ and in medical image analysis it is employed in 90 ' $\mathrm{s}^{[39]}$. Ivakhnenko et al. ${ }^{[40]}$ initially begin the deep leaning concept and later several works performed on deep leaning ${ }^{[41-43]}$. Some of the researchers applies the concept of deep learning in CBMIR system and achieved a good retrieval rate ${ }^{[44-46]}$. In ${ }^{[47]}$, a deep $\mathrm{CNN}$ which include five convolution layer is used for lungs pattern classification. Tulder ${ }^{[58]}$ proposed a convolutional restricted Boltzmann machine for classification of lung texture and detection of airways in CT images. For the segmentation of brain MRI, a CNN based method is introduced in ${ }^{[49]}$. Esteva et al. ${ }^{[50]}$ proposed a system for classification of skin cancer which is based on deep CNN Then, the CBMIR also used the concept of deep learning and proposed some biomedical image retrieval systems $^{[51-53]}$.

In medicine, the native texture-based descriptors perform a vital role as robust unique visual features. At first, two valued local binary pattern (LBP) introduced by Ojala et al. ${ }^{[54]}$. Then, Tan and Triggs ${ }^{[55]}$ proposed a modified LBP which is a three valued pattern and is known as local ternary pattern (LTP). A new pattern called local quantized extrema patterns (LQEPs) is proposed by Roa and Rao ${ }^{[56]}$ which is based on local quantized pattern ${ }^{[54]}$ and directional local extrema pattern $^{[57]}$. Again Rao and Roa presented local mech quantized extrema patterns ${ }^{[59]}$ which combine the LQEP with color histogram (RGB) for catching the color information. Deep et al. ${ }^{[60]}$ proposed a new descriptor DLTerQEP with the concepts from LBP, LTP and LQEP for retrieval and indexing of biomedical images. Murala et $a l .{ }^{[61]}$ adopt the concept of binary wavelet transform $(B W T)^{[62-64]}$ and draw out the multi-resolution binary image for biomedical image retrieval. In the same year, with the concept of local tetra patterns $(\mathrm{LTrPs})^{[65]}$ they come up with a new algorithm. In LTrPs, the information 
is extracted from a pixel and its neighbors. Local ternary co-occurrence pattern (LTCoP) is developed by the Murala and $\mathrm{Wu}^{[65]}$ which is depends on gray values. Later they introduced two new descriptors named local mesh patterns ${ }^{[66]}$ and Gabor local mesh pattern. Based on the descriptor some more biomedical image retrieval systems $^{[67-69]}$ are proposed.

Greenspan et al. ${ }^{[70]}$ designed a biomedical image retrieval method based on Gaussian mixture model (GMM) and Kullback- Leubler (KL) framework. For biomedical database a retrieval system is proposed by Oberoi et al. ${ }^{[71]}$ which is based on Harr wavelet or Fourier descriptor. With the help of J48 decision tree classifier, medical image retrieval is done $\mathrm{in}^{[72]}$. Wavelet transformed base image signature is used for developing an image retrieval system ${ }^{[73]}$ for medicine. For the performance evaluation of the proposed system, experiments are conducted on a diabetic retinopathy dataset and a mammography dataset. With scale invariant feature transform (SIFT) and LBP, ${ }^{[74]}$ perform the retrieval and classification of x-ray images. Many other concepts are also taken from the different methodologies to develop several biomedical retrieval systems ${ }^{[75-84]}$.

\section{Content Based Medical Image Retrieval (CBMIR)}

Initially, biomedical images from the training dataset are given as input to the proposed CBMIR system and then, the image is processed through the feature extraction step of the proposed system. In feature extraction step, HOG features are extracted from the input biomedical image which counts occurrences of gradient orientation in localized portions of an image and edge-based shape information from the input image is computed using canny edge detection algorithm which is a multi-step method which determines the edges and also suppress the noise. This generated feature vector represents the input biomedical image. PCA is employed over the generated feature vector to reduce the dimensionality. Similarly, a feature vector is computed for all the input biomedical training images and with these feature vector, the training database is formed which later used for the retrieval of related biomedical images.

After the formation of training database, testing is performed based on this database. The input query image is processed through the feature extraction and feature vector is computed for the given input query image. The query image is compared with the all other images stored at training dataset. With the help of euclidean distance, the distance between the query image feature vector and other feature vectors in the training dataset is computed to achieve the retrieval of top $\mathrm{n}$ related biomedical images.

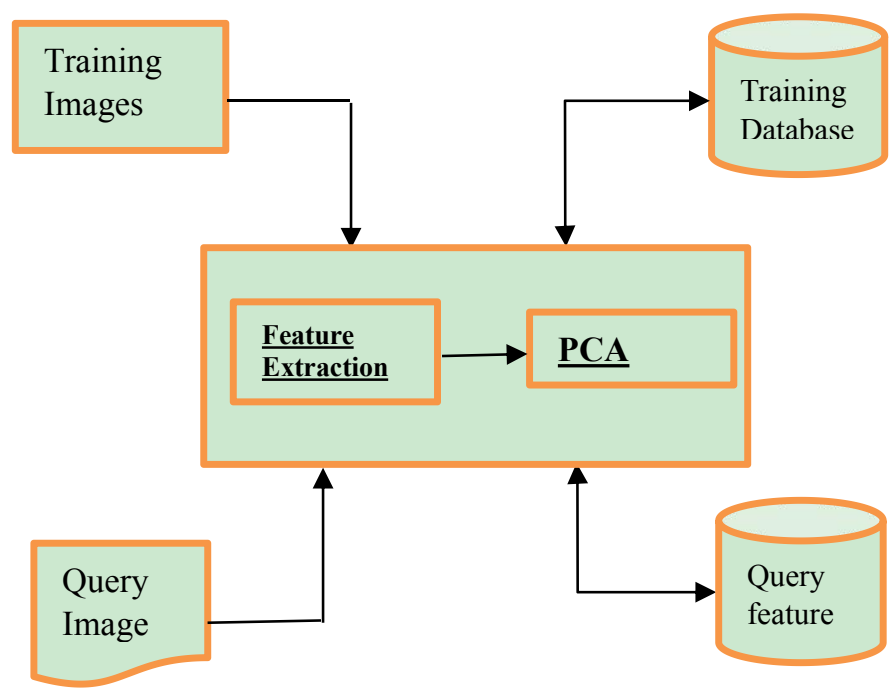

Figure 1; Framework for the proposed CBMIR system.

Figure 1 explains the framework of proposed CBMIR system and the algorithm for the proposed method is shown below:

\section{Algorithm:}

1. Load the input gray scale biomedical image.

2. Compute HOG features.

3. Locate and determine edge pixels using canny edge detection

4. Generate feature vector by integrating $\mathrm{HOG}$ feature and canny edge information.

5. Employ PCA over the feature vector.

6. Compute distance of query image from all other images in the training database.

7. Retrieve the related biomedical images based on shortest distance

\section{Results and disscussion}

To obtain the result, an image from the database is given as input query to the retrieval system and processed through each step and finally compare with all the images in the database by computing distances. Then, 
with the retrieved images the precision and recall is computed. Likewise, each image from the database is passed as query image to the system for computing the precision and recall. The precision of some query images for the proposed CBMIR system is shown on Table 1 and Table 2 shows the recall values obtained from the some of the query images. The performance of the proposed methodology is evaluated in ARP and ARR. Table 3 summarizes the performance of different methods in terms of ARP and Table 4 show the performance in terms of ARR.

\begin{tabular}{ccccccccccc} 
Query Image & $\mathbf{1}$ & $\mathbf{2}$ & $\mathbf{3}$ & $\mathbf{4}$ & $\mathbf{5}$ & $\mathbf{6}$ & $\mathbf{7}$ & $\mathbf{8}$ & $\mathbf{9}$ & $\mathbf{1 0}$ \\
\hline Precision (\%) & 87.50 & 83.33 & 81.81 & 76.92 & 83.33 & 90.90 & 71.42 & 100 & 87.50 & 100
\end{tabular}

Table 1. Performance of proposed CBMIR system in terms of precision values for some query image on lungs HRCT database

\begin{tabular}{lllllllllll} 
Query Image & $\mathbf{1}$ & $\mathbf{2}$ & $\mathbf{3}$ & $\mathbf{4}$ & $\mathbf{5}$ & $\mathbf{6}$ & $\mathbf{7}$ & $\mathbf{8}$ & $\mathbf{9}$ & $\mathbf{1 0}$ \\
\hline Recall (\%) & 70 & 100 & 90 & 100 & 100 & 100 & 100 & 80 & 70 & 90 \\
\hline
\end{tabular}

Table 2. Performance of proposed CBMIR system in terms of recall values for some query image on lungs HRCT database

\begin{tabular}{llllll} 
Methods & LBP & LBPu2_16_2 & LMePu2_16_2 & GLMePu2_8_1 & Proposed method \\
\hline ARP (\%) & 46.65 & 48.96 & 52.71 & 58.56 & 85.84
\end{tabular}

Table 3. Proposed method is compared with other existing methods in terms of ARP on lungs HRCT database

\begin{tabular}{llllll} 
Methods & LBP & LBPu2_16_2 & LMePu2_16_2 & GLMePu2_8_1 & Proposed method \\
\hline ARR (\%) & 55.35 & 57.27 & 58.00 & 61.33 & 90.20 \\
\hline
\end{tabular}

Table 4. Proposed method is compared with other existing methods in terms of ARR on lungs HRCT database

\section{Conclusion}

This paper presents an effective biomedical image retrieval system based on HOG features which counts occurrences of gradient orientation in localized portions of an image, edge based shape information using canny edge detection. To analyze the performance of the proposed CBMIR system, experiments are conducted on an ILD benchmark biomedical image database ${ }^{[85]}$ and the results are obtained in terms of precision, ARP, recall and ARP. An encouraging result is achieved which showns a substantial improvement as compared to LBP, LBPu2_16_2, LMePu2_16_2 and GLMePu2_8_1. This work is going to be helpful in alternative applications of image retrieval and indexing.

\section{References}

1. W.G. Bradley, "History of medical imaging", Proc Am Philos Soc, 2008, 152(3), pp. 349-361.

2. "History of the AIUM". Archived from the original on November 3, 2005. Retrieved November 15, 2005.

3. "The History of Ultrasound: A collection of recollections, articles, interviews and images". ww.obgyn.net. Archived from the original on 5 August 2006. Retrieved 2006-05-11

4. H.K. Huang, S.J. Dwyer, W. M. Angus, M.P. Capp, R.L. Arenson and H. Kangarloo, "Picture archiving and communications systems (PACS)". In: Radiological Society of North America $73^{\text {rd }}$ scientific assembly and annual meeting (Abstracts), 1987.

5. H.D. Fisher, K.M. McNeil, R. Vercillo and R.D. Lamoreaux, U.S. Patent No. 4,833,625. U.S. Patent 
and Trademark Office, Washington, DC, 1989.

6. P. Archiving P, Communication system. Fijifilm Medical Systems, USA, 1991.

7. C.R. Shyu, C. E. Brodley, A. C. Kak, A. Kosaka, A.M. Aisen and L.S. Broderick, "ASSERT: a physician-in-the-loop content-based retrieval system for HRCT image databases", Comput Vis Image Underst, Vol. 75(1-2), 1999, pp. 111-132.

8. D. Keysers, H. Ney, B.B. Wein and T.M. Lehmann, "Statistical framework for model-based image retrieval in medical applications. J Electron Imaging, Vol. 12(1),2003, pp. 59-68

9. M.O. Lam, T. Disney, D.S Raicu, J. Furst and D. S. Channin, "BRISC - an open source pulmonary nodule image retrieval framework", J Digit Imaging, Vol. 20(1), 2007, pp. 63-71.

10. T. Deselaers, D. Keysers and H. Ney, "FIRE-flexible image retrieval engine: ImageCLEF 2004 evaluation", In CLEF, 2004, pp 688-698.

11. H. Müller, N. Michoux and D. Bandon, A. Geissbuhler, "A review of content-based image retrieval systems in medical applications - clinical benefits and future directions", Int J Med Inform, Vol. 73(1), 2003, pp. $1-23$.

12. K. H. Hwang, H. Lee and D. Choi, "Medical image retrieval: past and present", Healthcare Inform Res, Vol. 18(1), 2012, pp. 3-9.

13. P. Das and A. Neelima, "An overview of approaches for content-based medical image retrieval", Int J Multimed Info Retr, Vol. 6(4), 2017, pp. 271-280.

14. P. Ghosh, S. Antani, L.R. Long and G.R. Thoma, "Review of medical image retrieval systems and future directions", In: 2011 24th international symposium on computer-based medical systems (CBMS), IEEE, 2011, pp 1-6.

15. X.S. Zhou and T.S. Huang, "Relevance feedback in image retrieval: a comprehensive review", Multimed Syst, vol. 8(6), 2003, pp. 536-544.

16. C.B. Akgül, D.L.Rubin, S. Napel, C.F. Beaulieu, H. Greenspan and B. Acar, "Content-based image retrieval in radiology: current status and future directions", J Digit Imaging, Vol. 24(2),2011, pp. 208-222.

17. A. Kumar, J. Kim, W. Cai, M. Fulham and D. Feng, "Content-based medical image retrieval: a survey of applications to multidimensional and multimodality data", J Digit Imaging, Vol. 26(6), 2013, pp. 1025-1039.

18. M. Rehman, M. Iqbal, M. Sharif and M. Raza, "Content based image retrieval: survey", World Appl Sci J, Vol. 19(3), 2012, pp. 404-12.

19. G. Deep, L. Kaur and S. Gupta, "Biomedical image indexing and retrieval descriptors: a comparative study", Procedia Comput Sci, Vol. 85, 2016, pp. 954-961.

20. H.R. Tizhoosh, "Barcode annotations for medical image retrieval: a preliminary investigation", In: 2015 IEEE international conference on image processing (ICIP), IEEE, 2015, pp 818-822.

21. H.R. Tizhoosh, M. Gangeh, H. Tadayyon and G. J. Czarnota, "Tumour ROI estimation in ultrasound images via radon barcodes in patients with locally advanced breast cancer", In: 2016 IEEE $13^{\text {th }}$ international symposium on biomedical imaging (ISBI), IEEE, 2016, pp 1185-1189.

22. H.R. Tizhoosh, S. Zhu, H. Lo, V. Chaudhari and T. Mehdi, "MinMax radon barcodes for medical image retrieval", In: International symposium on visual computing. Springer International Publishing, 2016, pp 617-627.

23. H.R. Tizhoosh, C. Mitcheltree, S. Zhu and S. Dutta, "Barcodes for medical image retrieval using autoencoded radon transform", In: 2016 23rd international conference on pattern recognition (ICPR), IEEE, 2016, pp 3150-3155.

24. M. Nouredanesh, H.R. Tizhoosh, E. Banijamali and J. Tung, "Radon-Gabor barcodes for medical image retrieval", In: $201623^{\text {rd }}$ international conference on pattern recognition (ICPR), IEEE, 2016, pp 1309-1314.

25. M. Babaie, H.R. Tizhoosh, S. Zhu and M.E. Shiri, "Retrieving similar X-ray images from big image data using radon barcodes with single projections", 2017, arXiv preprint arXiv:1701.00449.

26. M.K. Kundu, M. Chowdhury, S. Das, "Interactive radiographic image retrieval system", Comput Methods Programs Biomed, Vol. 139, 2017, pp. 209-220.

27. L.Ma, X. Liu, Y. Gao, Y. Zhao, X. Zhao and C. Zhou, "A new method of content based medical image retrieval and its applications to $\mathrm{CT}$ imaging sign retrieval", J Biomed Inform, Vol. 66, 2017, pp.148-158.

28. J. Nowaková, M. Prílepok and V. Snášel, "Medical image retrieval using vector quantization and fuzzy S-tree”, J Med Syst, Vol. 41(2), 2017, pp. 18.

29. Chatzichristofis SA, Boutalis YS (2010) Content based radiology image retrieval using a fuzzy rule based scalable composite descriptor. Multimed Tools Appl 46(2-3):493-519

30. G. Zhang, Z.M. Ma, "Texture feature extraction and description using Gabor wavelet in content-based medical image retrieval", In: ICWAPR'07, International conference on wavelet analysis and pattern recognition, IEEE, Vol. 1., 2007, pp 169-173.

31. K. Fukushima, "Neocognitron: a self-organizing neural network model for a mechanism of pattern recognition unaffected by shift in position", BiolCybern, Vol. 36(4), 1980, pp. 93-202.

32. K. Fukushima and S. Miyake, "Neocognitron: a self-organizing neural network model for a mechanism of visual pattern recognition", In: van Hemmen JL (ed) Competition and cooperation in 
neural nets. Springer, Berlin, 1982, pp 267-285.

33. K. Fukushima and S. Miyake, "Neocognitron: a new algorithm for pattern recognition tolerant of deformations and shifts in position", Pattern Recognit, Vol. 15(6), 1982, pp. 455-469.

34. K. Fukushima, S. Miyake and T. Ito, "Neocognitron: a neuralnetwork model for a mechanism of visual pattern recognition", IEEE Trans Syst Man Cybern, Vol. 5, 1983, pp. 826-834.

35. K. Fukushima, "A neural network model for selective attention in visual pattern recognition", Biol Cybern, Vol. 55(1), 1986, pp. 5-15.

36. K. Fukushima, "Neural network model for selective attention in visual pattern recognition and associative recall”, Appl Opt, Vol. 26(23), 1987, 4985-92.

37. K. Fukushima, "Neocognitron: a hierarchical neural network capable of visual pattern recognition", Neural Netw, Vol. 1(2), 1988, pp. 119-130.

38. Fukushima K, "A neural network for visual pattern recognition", Computer, Vol. 21(3), 1988, pp. 65-75.

39. S.C. Lo, S.L. Lou, J.S. Lin, M.T. Freedman, M.V. Chien and S.K. Mun, "Artificial convolution neural network techniques and applications for lung nodule detection", IEEE, Trans Med Imaging, Vol. 14(4), 1995, pp.711-718.

40. A.G. Ivakhnenko and V.G. Lap, "Cybernetic predicting devices", CCM Information Corporation, 1965.

41. R.H. Hahnloser, R. Sarpeshkar, M.A. Mahowald, R.J. Douglas and H.S. Seung, "Digital selection and analogue amplification coexist in a cortex-inspired silicon circuit", Nature, Vol. 405(6789), pp. 947.

42. X. Glorot, A. Bordes and Y. Bengio, "Deep sparse rectifier neural networks", In: Proceedings of the fourteenth international conference on artificial intelligence and statistics, 2011, pp 315-323.

43. X. Glorot and Y. Bengio, "Understanding the difficulty of training deep feedforward neural networks", In: Proceedings of the thirteenth international conference on artificial intelligence and statistics, 2010, pp. 249-256.

44. J. Wan, D. Wang, S.C.H. Hoi, P. Wu, J. Zhu, Y. Zhang and $\mathrm{J}$. Li, "Deep learning for content-based image retrieval: a comprehensive study", In: Proceedings of the 22nd ACM international conference on multimedia, 2014, pp 157-166.

45. A. Babenko and V. Lempitsky, "Aggregating local deep features for image retrieval", In: Proceedings of the IEEE international conference on computer vision, 2015, pp 1269-1277.

46. K. Lin, H.F. Yang, J.H. Hsiao and C.S. Chen, "Deep learning of binary hash codes for fast image retrieval", In: Proceedings of the IEEE conference on computer vision and pattern recognition workshops, 2015, pp 27-35.

47. M. Anthimopoulos, S. Christodoulidis, L. Ebner, A.
Christe and S. Mougiakakou, "Lung pattern classification for interstitial lung diseases using a deep convolutional neural network", IEEE Trans Med Imaging, Vol. 35(5), 2016, pp. 1207-1216.

48. G. Van Tulder and M. de Bruijne, "Combining generative and discriminative representation learning for lung CT analysis with convolutional restricted Boltzmann machines", IEEE Trans Med Imaging, Vol. 35(5), 2016, pp. 1262-1272.

49. P. Moeskops, M.A Viergever, A.M. Mendrik, L.S. de Vries, M.J. Benders and I. Išgum, "Automatic segmentation of MR brain images with a convolutional neural network", IEEE Trans Med Imaging, Vol. 35(5), 2016, pp. 1252-1261.

50. A. Esteva, B. Kuprel, R.A Novoa, J. Ko, S.M. Swetter, H.M. Blau and S. Thrun, "Dermatologist-level classification of skin cancer with deep neural networks", Nature, Vol. 542(7639), 2017, pp. 115-118.

51. Y. Cao, S. Steffey, J. He, D. Xiao, C. Tao, P. Chen and H. Müller, "Medical image retrieval: a multimodal approach”, Cancer Inform 13(Suppl 3), 2014, pp.125.

52. Q. Sun, Y. Yang, J. Sun, Z. Yang and J. Zhang, "Using deep learning for content-based medical image retrieval", In: SPIE medical imaging. International Society for Optics and Photonics, 2017, pp 1013812-1013812.

53. A. Qayyum, S.M. Anwar, M. Awais and M. Majid, "Medical image retrieval using deep convolutional neural network", Neurocomputing, Vol. 266, 2017, pp. $8-20$.

54. T. Ojala, M. Pietikäinen and D. Harwood, "A comparative study of texture measures with classification based on featured distributions", Pattern Recognit, Vol. 29(1), 1996, pp. 51-59.

55. X. Tan and B. Triggs, "Enhanced local texture feature sets for face recognition under difficult lighting conditions", IEEE Trans Image Process, Vol. 19(6), 2010, pp. 1635-1650.

56. S. ul Hussain and B. Triggs, "Visual recognition using local quantized patterns", In: Computer vision-ECCV 2012, Springer, Berlin, 2012, pp 716-729.

57. S. Murala, R.P. Maheshwari and R. Balasubramanian, "Directional local extrema patterns: a new descriptor for content based image retrieval", Int J Multimed Inf Retr, Vol. 1(3), 2012, pp. 191-203.

58. L.K. Rao and D.V. Rao, "Local quantized extrema patterns for content-based natural and texture image retrieval", Hum Centric Comput Inf Sci, Vol. 5(1), 2015, pp. 26.

59. L.K. Rao, D.V. Rao and L.P. Reddy, "Local mesh quantized extrema patterns for image retrieval", SpringerPlus, Vol. 5(1), 2016, pp. 1-15.

60. G. Deep, L. Kaur and S. Gupta, "Directional local ternary quantized extrema pattern: a new descriptor for biomedical image indexing and retrieval", Eng 
Sci Technol Int J, Vol. 19(4), 2016, pp.1895-1909.

61. S. Murala, R.P. Maheshwari and R. Balasubramanian, "Directional binary wavelet patterns for biomedical image indexing and retrieval", J Med Syst, Vol. 36(5), 2012, pp. 2865-2879.

62. M.D. Swanson and A.H Tewfik, "A binary wavelet decomposition of binary images", IEEE Trans Image Process, Vol. 5(12), pp.1637-1650.

63. L. Kamstra, "The design of linear binary wavelet transforms and their application to binary image compression", In: 2003 Proceedings International conference on image processing, IEEE, Vol 3., pp III-241.

64. H. Pan, L.Z. Jin, X.H. Yuan, S.Y. S Xia and L.Z. $\mathrm{Xia}$, "Context-based embedded image compression using binary wavelet transform", Image Vis Comput, Vol. 28(6), 2010, pp. 991-1002.

65. S. Murala, R.P. Maheshwari and R. Balasubramanian, "Local tetra patterns: a new feature descriptor for content-based image retrieval", IEEE Trans Image Process, Vol. 21(5), 2012, pp. 2874-2886.

66. S. Murala and Q. J. Wu, "Local mesh patterns versus local binary patterns: biomedical image indexing and retrieval", IEEE J Biomed Health Inform, Vol. 18(3), 2014, pp. 929-938.

67. A. Lumini, L. Nanni and S. Brahnam, "Multilayer descriptors for medical image classification", Comput Biol Med, Vol. 72, 2016, pp. 239-247.

68. V. Ojansivu and J. Heikkilä, "Blur insensitive texture classification using local phase quantization", In: International conference on image and signal processing, Springer, Berlin, 2008, pp. 236-243.

69. S. Murala and Q. J. Wu, "Spherical symmetric 3D local ternary patterns for natural, texture and biomedical image indexing and retrieval", Neurocomputing, Vol. 149, 2015, pp. 1502-1514.

70. H. Greenspan and A.T. Pinhas, "Medical image categorization and retrieval for PACS using the GMM-KL framework", IEEE Trans Inf Technol Biomed, Vol. 11(2), 2007, pp. 190-202.

71. A. Oberoi and M. Singh, "Content based image retrieval system for medical databases (CBIR-MD)-lucratively tested on endoscopy, dental and skull images", IJCSI Int J Comput Sci Issues 9(3), 2012, pp. 1694-1814.

72. A.N. Krishna and B.G Prasad BG, "Automated image annotation for semantic indexing and retrieval of medical images", Int J Comput Appl,Vol. 55(3), 2012, pp.26-33.

73. G. Quellec, M. Lamard, G. Cazuguel, B. Cochener and C. Roux, "Wavelet optimization for content-based image retrieval in medical databases", Med Image Anal, Vol. 14(2), 2010, pp. 227-241.

74. M.R. Zare and H. Müller, "A medical X-ray image classification and retrieval system", In: PACIS,
2016, pp. 13.

75. M.M. Rahman, S.K. Antani and G.R. Thoma, "A learning-based similarity fusion and filtering approach for biomedical image retrieval using SVM classification and relevance feedback", IEEE Trans Inf Technol Biomed, Vol. 15(4), 2011, pp. 640-646.

76. M.M. Rahman, S.K. Antani and G.R. Thoma, "A medical image retrieval framework in correlation enhanced visual concept feature space", In: 22nd IEEE international symposium on computer-based medical systems, CBMS 2009, 2009, IEEE, pp. 1-4.

77. M.M. Rahman, P. Bhattacharya and B.C Desai, "A framework for medical image retrieval using machine learning and statistical similarity matching techniques with relevance feedback", IEEE Trans Inf Technol Biomed, Vol. 11(1), 2007, pp. 58-69.

78. M.R. Nazari, E. Fatemizadeh, "A CBIR system for human brain magnetic resonance image indexing", Int J Comput Appl, Vol. 7(14), 2010, pp. 33-37.

79. I.F. Amaral, F. Coelho, J.F.P. da Costa and J.S. Cardoso, "Hierarchical medical image annotation using SVM-based approaches", In: 2010 10th IEEE international conference on information technology and applications in biomedicine (ITAB). IEEE, 2010, pp. 1-5.

80. C. Lacoste, J.P. Chevallet, J.H. Lim, X. Wei, D. Racoceanu, DTH Le and N. Vuillenemot, IPAL knowledge-based medical image retrieval in ImageCLEFmed 2006. In: CLEF (Working Notes).

81. C. Lacoste, J.P. Chevallet, J.H Lim, DTH Le, W. Xiong, D. Racoceanu and N. Vuillenemot, "Inter-media concept-based medical image indexing and retrieval with umls at IPAL", In: Workshop of the cross-language evaluation forum for European languages. Springer, Berlin, pp 694-701.

82. J.H. Lim and J.P. Chevallet JP, Vismed: a visual vocabulary approach for medical image indexing and retrieval, Inf Retr Technol, Part of Lecture Notes in Computer Science book series LNCS, vol 3689, 2005, pp 84-96.

83. C. Lacoste, J.H Lim, J.P Chevallet and DTH Le, "Medical-image retrieval based on knowledge-assisted text and image indexing", IEEE Trans Circuits Syst Video Technol, 17(7), 2007, 889-900.

84. J.H. Lim, J.P. Chevallet, DTM Le, H Goh, "Bi-modal conceptual indexing for medical image retrieval. In: International conference on multimedia modeling”, Springer Berlin, 2008, pp. 456-465.

85. A. Depeursinge, A. Vargas, A. Platon, A. Geissbuhler, P.A. Poletti and H. Müller, "Building a reference multimedia database for interstitial lung diseases", In: Computerized Medical Imaging and Graphics, Vol. 36, 2011, pp. 3(227-238). 